\title{
Static Analysis of Scale Model for Ribbed Ring Stiffened Shallow Spherical Shell
}

\author{
Jianwen Liu', a, Dongyun Jia ${ }^{1, b}$ and Zhan Gao ${ }^{1, c}$ \\ ${ }^{1}$ Anhui University of Technology, Ma'anshan, China; \\ a15855636700@163.com, bgloriajia07@163.com, c18395561639@163.com
}

Keywords: Shallow spherical shell, Static analysis, Scale model, Ribbed ring, Gasholder Abstract. The structure of large ribbed ring stiffened shallow spherical shell is widely used for roof design of gasholders and oil storage tanks. The FEM of scale shell structure is established and the static performance is analyzed. The grid verification and time steps verification are performed to assure the accuracy of the calculation. The research about static performance shows the yield order of components. The elastic critical load values are obtained from the load-displacement curves of the components. Analysis results can be referred for subsequent model testing and engineering design.

\section{Introduction}

Storage tank structures are developing towards large capacity, high pressure, and large scale $\mathrm{e}^{[1]}$. Ribbed ring stiffened shallow spherical shell structure can meet the requirements of large span, high bearing capacity and easy production and installation. Many large-scale roofs that exceed the relevant regulations of the storage tanks have been built, which brings great security risks into production ${ }^{[2]}$. The finite element analysis can reveal the mechanism of the ribbed ring shell structure ${ }^{[3,4]}$. Destructive testing of actual projects does not meet economic requirements. The scale model of the ribbed ring stiffened shallow spherical shell is established and analyzed to explore the mechanism of failure under static loading. The results can be applied to compare with testing results.

\section{The design of scale model}

The ribbed ring shell of a $60 \mathrm{~m}$ diameter is taken as the research object. The rise span ratio of the shell is $1: 10$. The main ribs are channel steel [18a. The radial secondary rib is angle steel L75, the thickness of the top plate is $6 \mathrm{~mm}$. The center of the shell has a $3 \mathrm{~m}$ diameter hole. There are 24 radial main ribs and 8 circumferential main ribs. The radial secondary ribs are arranged in the unit enclosed by the radial and circumferential skeletons. From the first ring belt (outer circle) to the center ring belt, the quantities of radial secondary ribs are $5,4,3,3,2,2,1$, and 0 . The structure sketch is shown in Figure 1.

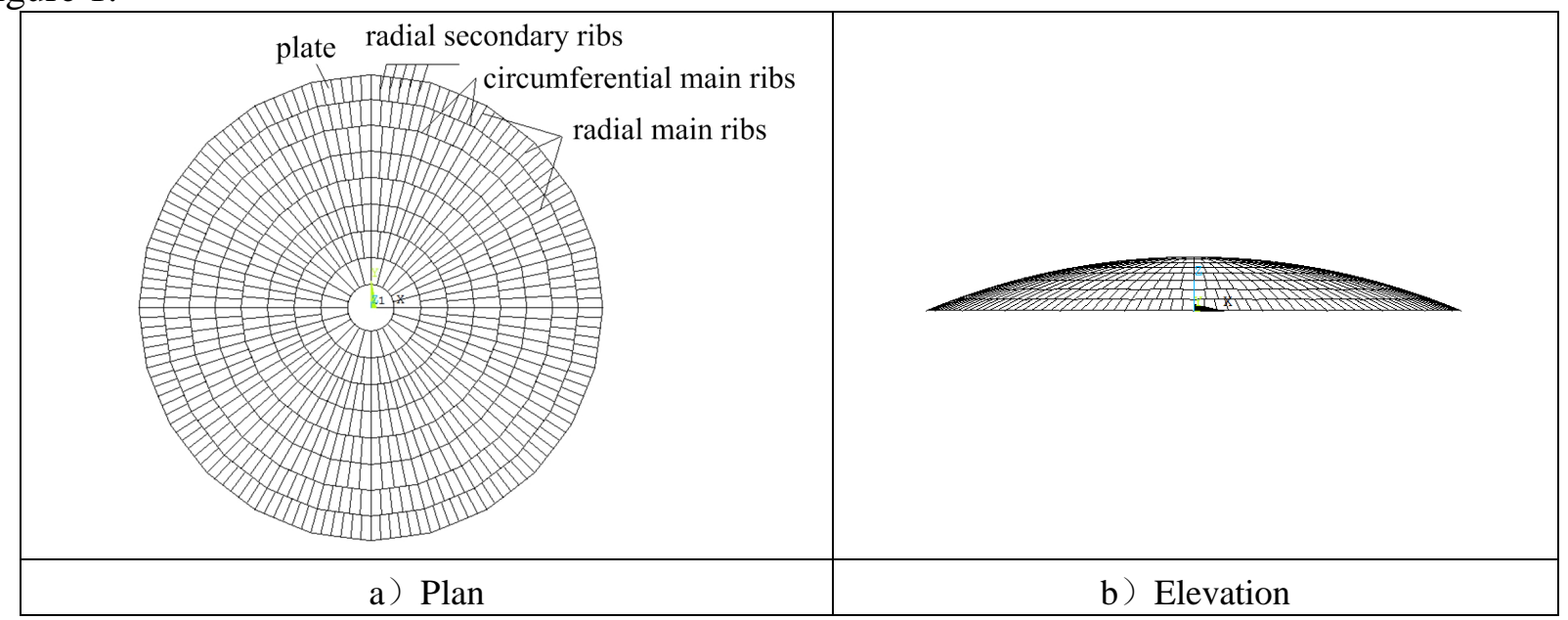

Fig.1. The ribbed ring stiffened shallow spherical shell

Based on the manufacture conditions and the thickness of thin plate, the FEM is scaled at ratio of 1:20,and the sections of channel steel and angle steel are simplified to thin plates. Compared with the 
actual structure, the simplified scale model must be satisfied the similarity ratio requirements ${ }^{[5]}$. The channel steel [18a, angle steel L75 and $6 \mathrm{~mm}$ plate are simplified and scaled to $1.3 \mathrm{~mm} \times 6 \mathrm{~mm}$, $0.4 \mathrm{~mm} \times 6 \mathrm{~mm}$ and $0.3 \mathrm{~mm}$ respectively. In order to ensure that the cross-section of the scale model similar to actual structure both in radial bending stiffness and in tensile compression stiffness, a computing unit is taken as shown in Figure 2. The combined section properties of area and moment of inertia are compared between the actual structure, the scale structure and the simplified scale structure as shown in Table 1.

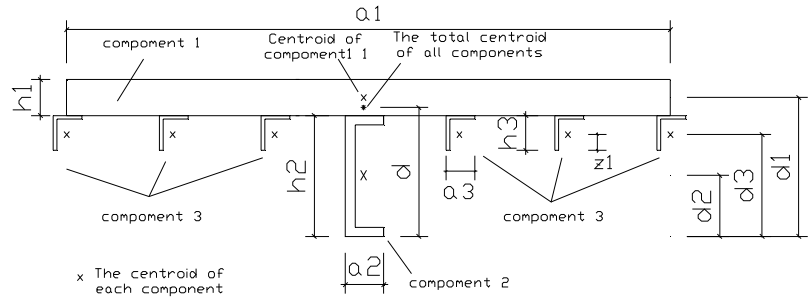

a) Actual section

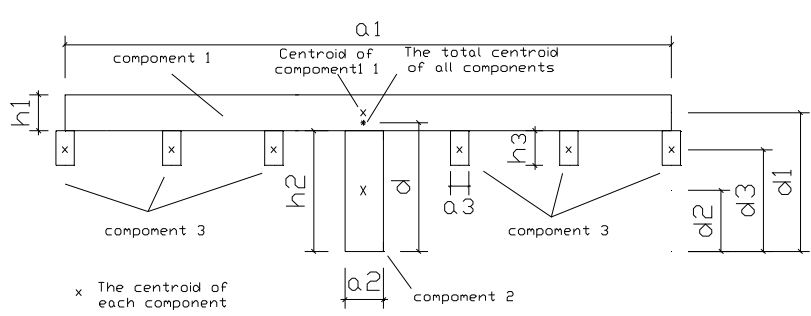

b) Simplified scale section

Fig.2. Combination section of a computing unit

Table 1 The comparison of section properties

\begin{tabular}{cccc}
\hline & Actual section & Scale section & Simplified scale section \\
\hline Area A $\left(\mathrm{mm}^{2}\right)$ & 43851.4 & 109.63 & 137.55 \\
Moment of inertia I $\left(\mathrm{mm}^{4}\right)$ & 37202570 & 232.5 & 228.5 \\
\hline
\end{tabular}

\section{Static Analysis of Simplified Scale Model}

Grid validation of the model In order to ensure the accuracy of the model analysis results, the density of the mesh needs to be compared and verified. Generally, the greater the density of the grid, the closer the accuracy of analysis, the longer the time required for the calculation, and the higher the cost of the calculation. To obtain a more accurate solution within a reasonable solution time, the optimum density range of the grid needs to be found. Therefore, the static load, under which the shell is yielding, is compared with different grid. Comparison of grid density and analysis results is shown in Figure 3.

From Figure 3, it can be found: to archive the $1.6 \mathrm{~mm}$ displacement of the stiffened shell, the static load reaches $25 \mathrm{kpa}$ with $0.03 \mathrm{~m}$ grid , the static load increases to $25.2 \mathrm{kpa}$ with $0.02 \mathrm{~m}$ grid. The error is within $5 \%$. Therefore, a $0.03 \mathrm{~m}$ grid can be selected for simulation calculation. 


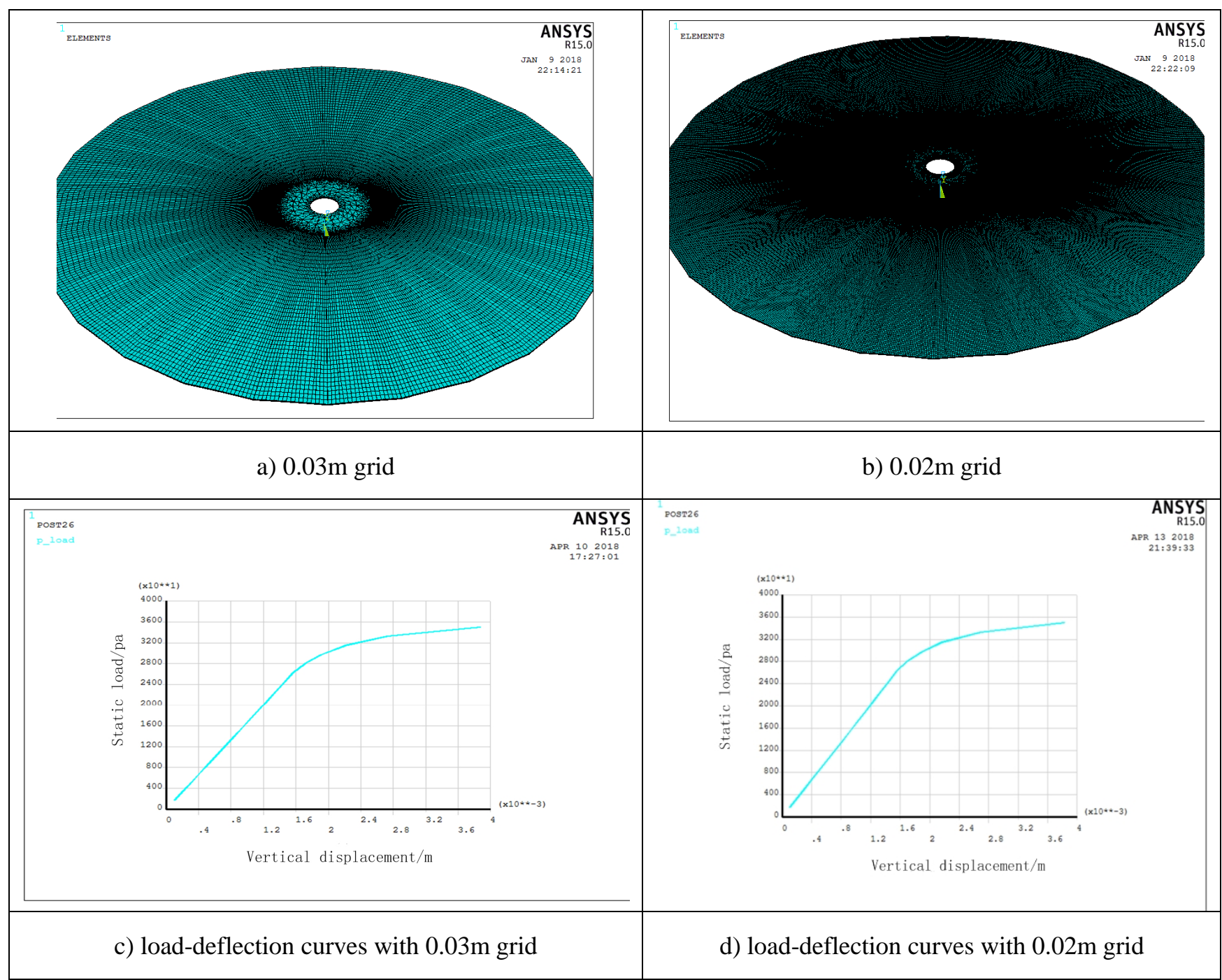

Fig.3. Comparison of grid density and analysis results

Time step verification of the model solution To ensure that the solution tends to convergence, a reasonable time step is to be set. The maximum displacements of the top shell are select from the load-displacement curve for comparison. The results of the iterations with 20 steps, 50 steps, 80 steps, and 100 steps are listed in Figure 4.

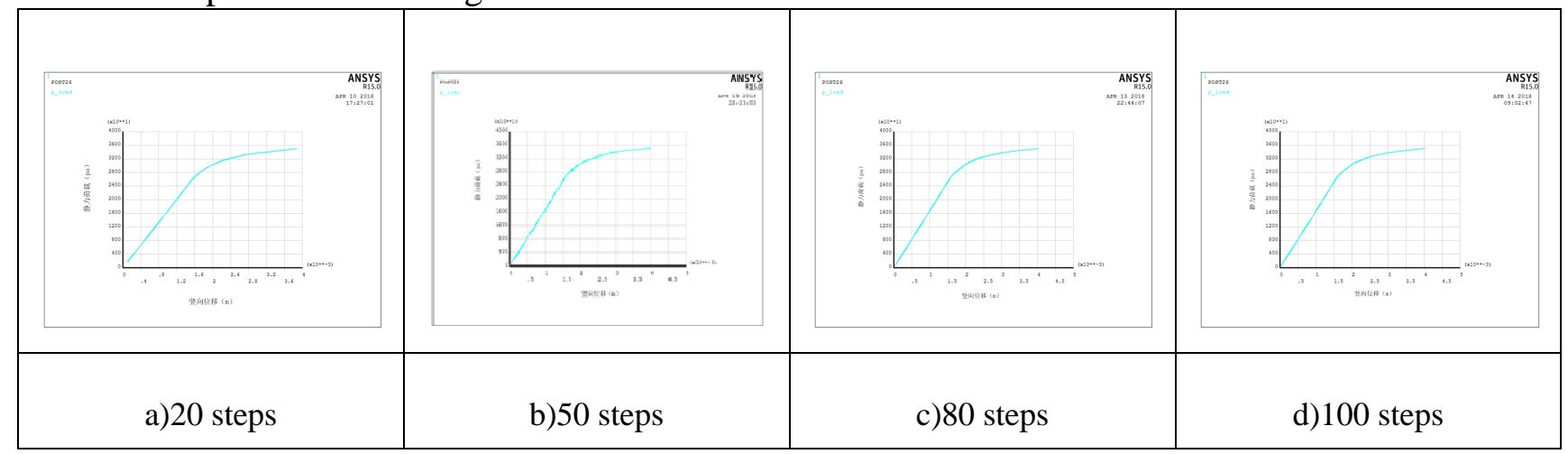

Fig.4. Load-displacement curves of maximum displacement with different iteration steps

It can be seen from Fig. 4 that the yield points for the time steps with 20 steps, 50 steps, 80 steps and 100 steps are all near $25 \mathrm{kpa}$. The error is within $5 \%$. Therefore, it is reasonable and effective to set the time step as 20 steps. 
Static performance analysis of the model Based on the grid and time step verification results, the static analysis of the simplified scale model is performed with a $0.03 \mathrm{~m}$ grid and 20 steps. The stress of the structure under uniform load is shown in Figure $5^{[6]}$.

It can be seen from Fig. 5 that: the yield zone starts from the first ring belt, and then the center ring beam. As the load increases, the yield zone spreads to the central belts. the entire shell yields until the load reaches $33.25 \mathrm{kpa}$.

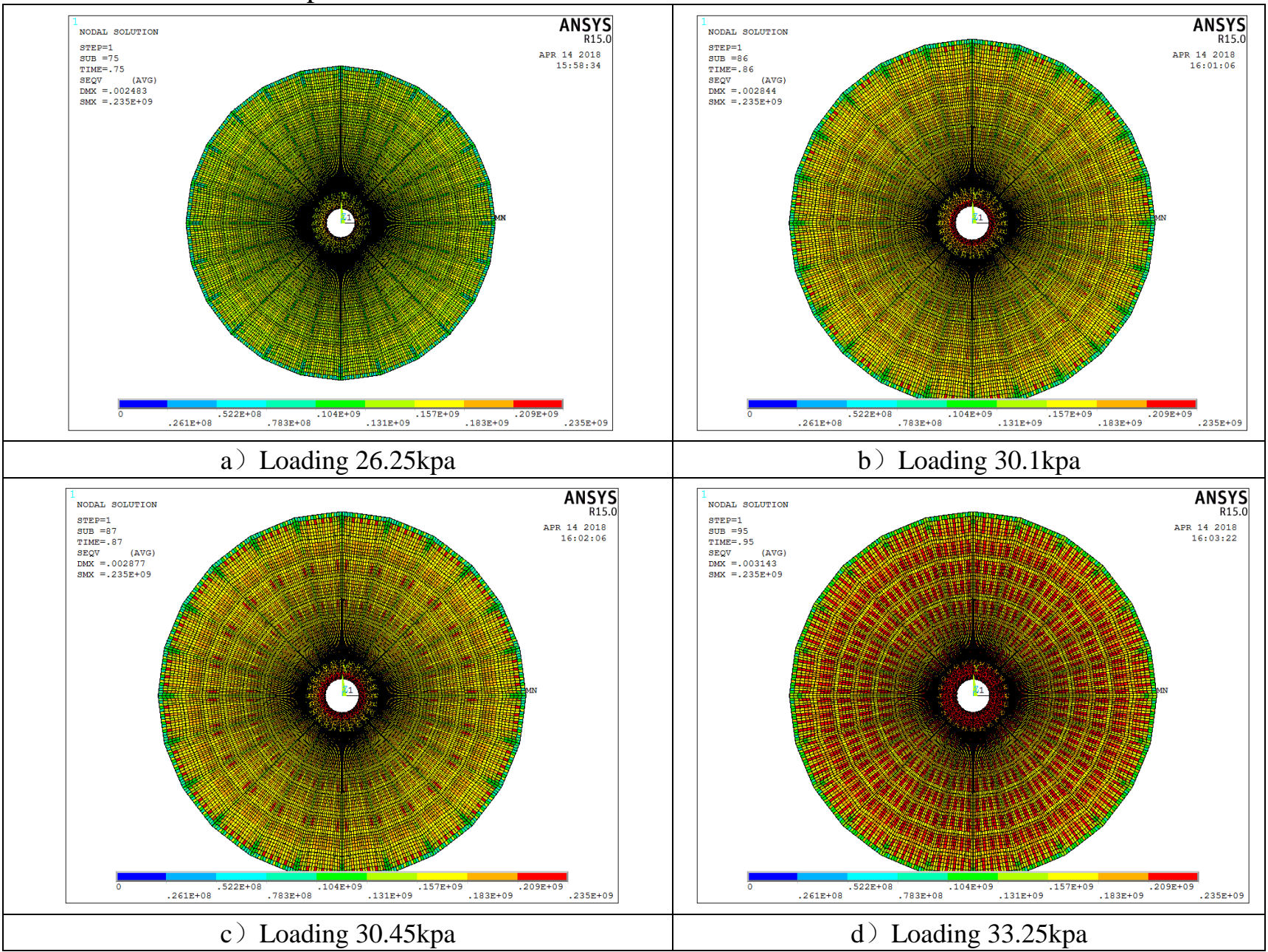

Fig.5. Structural stress diagram changes with load

The Load-displacement curves for the secondary rib, main rib at the 1st ring belt, the central ring beam and the shell are shown in Figure 6. At the turning point, aforementioned components reach the yield stress $235 \mathrm{Mpa}$. The loading values at the turning points of each curve are listed in Table 2.

\begin{tabular}{|c|c|c|c|}
\hline & center ring beam & shell \\
\hline $\begin{array}{c}\text { secondary rib } \\
\text { at the 1st ring belt }\end{array}$ & main rib \\
at the 1st ring belt
\end{tabular}

Fig.6. Load-displacement curves

Table.2. The load at the turning point of each curve（kpa）

\begin{tabular}{ccccc}
\hline & secondary rib & main rib & center ring beam & shell \\
\hline Load $(\mathrm{kpa})$ & 14.0 & 23.0 & 34.0 & 35.0 \\
\hline
\end{tabular}


The yield order of the structure components can be seen from Table 2. Based on the analysis results, the stress of ribs at the fifth and sixth belts is $205 \mathrm{Mpa}$ at the load of $35 \mathrm{kpa}$. And it can be considered that the first ring belt has destroyed at $35 \mathrm{kpa}$ loading.

\section{Conclusions}

1) Based on the principle of similarity ratio, A reasonable simplified scaled model of the stiffened shallow spherical shell is obtained.

2) In finite element analysis, the grid density and time step affect the accuracy of the calculation. The grid verification and time step verification are performed to assure correct analysis .

3 ) The order of yielding of structural members is found by static analysis. The components at first ring belt and the center ring beam are the key parts both in the structure design and in the model testing of the ribbed ring stiffened shallow spherical shell.

\section{Acknowledgements}

The completion of this paper thanks to the support of the Natural Science Foundation of Anhui Province, Fund number(1708085ME126).

\section{References}

[1] Dongyun Jia, Study on Static and Dynamic Performance for the Large-scale Regular Polygon Gasholder, PhD, Hohai University, Nanjing in China, 2010

[2] Jingbo Li, The Supergage of Ribbed Valut Storages Design, SHANDONG CHEMICAL INDUSTRY, Vol. 36 (2007), p. 23-24

[3] Xinmin Wang, in: ANSYS Numerical Analysis Of Engineering Structure, edited by Zhimin Chen, Structural Nonlinear Analysis, chapter, 8, China Communications Press(2007), (in chinese)

[4] Dejin Jiang, Xiaoyong Xu, Design of shallow spherical shell top cover in gas storage cabinet, JIANGSU STEEL STRUCTURE, vol. 5 (2011), p. 217-220

[5] Jiang Dejin, Yang Jun, Jia Dongyun, DISCUSSION ON SOME PROBLEMS IN THE DESIGN OF STEEL SHALLOW SPHERICAL SHELL, STEEL CONSTRUCTION, vol. 30(2015) p. $59 \sim 63$

[6] K.P.Buchert, in: Shell and Shell-like Structures Guide to Stability design Criteria for Metal Structures, DOUBLY CURVED SHELLS AND SHELL LIKE STRUCTURES, CHAPTER,18, P. 807-823(1976) 